\title{
treedata.table: A wrapper for data.table that enables fast manipulation of large phylogenetic trees matched to data
}

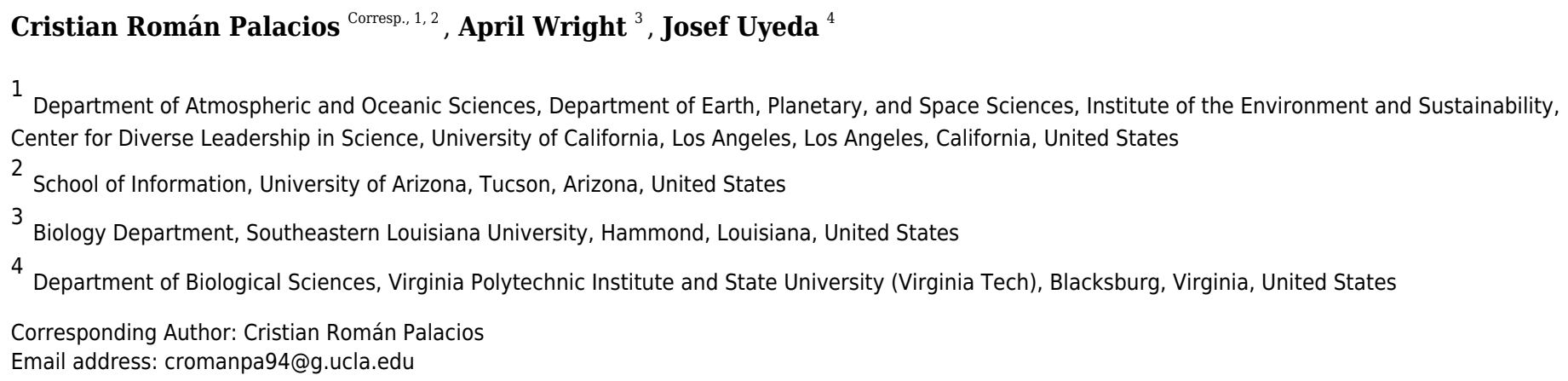

The number of terminals in phylogenetic trees has significantly increased over the last decade. This trend reflects recent advances in next-generation sequencing, accessibility of public data repositories, and the increased use of phylogenies in many fields. Despite $R$ being central to the analysis of phylogenetic data, manipulation of phylogenetic comparative datasets remains slow, complex, and poorly reproducible. Here, we describe the first R package extending the functionality and syntax of data.table to explicitly deal with phylogenetic comparative datasets. treedata.table significantly increases speed and reproducibility during the data manipulation steps involved in the phylogenetic comparative workflow in $\mathrm{R}$. The latest release of treedata.table is currently available through CRAN ( https://cran.r-project.org/web/packages/treedata.table/ ). Additional documentation can be accessed through rOpenSci ( https://ropensci.github.io/treedata.table/ ). 
1 treedata . table: A wrapper for data. table that enables fast

2 manipulation of large phylogenetic trees matched to data

3

4

5

6

7

8

Cristian Román-Palacios ${ }^{1,2, *}$, April M. Wright ${ }^{3}$, and Josef C. Uyeda ${ }^{4}$

${ }^{1}$ Department of Atmospheric and Oceanic Sciences, Department of Earth, Planetary, and Space Sciences, Institute of the Environment and Sustainability, Center for Diverse Leadership in Science, University of California - Los Angeles, Los Angeles, CA 90095 USA

2 School of Information, University of Arizona, Tucson, AZ, 85721, USA

${ }^{3}$ Department of Biological Sciences, Southeastern Louisiana University, Hammond, LA, 70403, USA

${ }^{4}$ Department of Biological Sciences, Virginia Tech, 926 West Campus Drive, Blacksburg, Virginia 24061, USA

Corresponding Author:

Cristian Román-Palacios ${ }^{1,2}$

520 Portola Plaza, Los Angeles, CA, 90095, USA

Email address: cromanpa@g.ucla.edu; cromanpa94@gmail.com

\section{Abstract}

The number of terminals in phylogenetic trees has significantly increased over the last decade. This trend reflects recent advances in next-generation sequencing, accessibility of public data repositories, and the increased use of phylogenies in many fields. Despite $R$ being central to the analysis of phylogenetic data, manipulation of phylogenetic comparative datasets remains slow, complex, and poorly reproducible. Here, we describe the first $\mathrm{R}$ package extending the functionality and syntax of data. table to explicitly deal with phylogenetic comparative datasets. treedata.table significantly increases speed and reproducibility during the data manipulation steps involved in the phylogenetic comparative workflow in $\mathrm{R}$. The latest release of treedata. table is currently available through CRAN (https://cran.rproject.org/web/packages/treedata.table/). Additional documentation can be accessed through rOpenSci (https://ropensci.github.io/treedata.table/).

\section{Introduction}

The number and size of published phylogenetic trees have exponentially increased over the years (Fig. 1; Smith et al., 2011; FitzJohn et al., 2014; Smith \& Brown, 2019). Ongoing biodiversity sequencing efforts have triggered the development of phylogenetic computational methods able to deal with datasets involving hundreds of thousands of 
41 taxa (McMahon et al., 2015). For instance, the early development of MAFFT (Katoh

42 2002) significantly decreased computational times required to perform sequence

43 alignment on molecular datasets with thousands of species. Similarly, RAxML

44 (Stamatakis, 2006), PATHd8 (Tamura et al., 2012), and TreePL (Smith \& O'Meara, 45 2012) greatly reduced computational times during the inference and absolute dating of phylogenetic trees including thousands of species. Given the unprecedented pace at which phylogenetic data is accumulating (Piel et al., 2000; Redelings \& Holder, 2017), updating the current comparative phylogenetic workflow to cope with the increasing size of phylogenetic trees is now more critical than ever. Attention should be paid to the development of faster, computationally efficient, and more user-friendly implementations in $\mathrm{R}$ that further increase reproducibility. The $\mathrm{R}$ language ( $\mathrm{R}$ Development Core Team, 2013) is now central to research utilizing phylogenetic comparative methods, and many essential packages and educational materials are made available using this language (Harmon, 2019). The latest release of treedata. table is available through CRAN (https://cran.r-project.org/web/packages/treedata.table/). More information about the treedata.table $\mathrm{R}$ package can be found in rOpenSci

(https://ropensci.github.io/treedata.table/).

58

\section{A short description of data. table}

treedata. table heavily relies on data. table, an $\mathrm{R}$ package that enables highperformance extended functionality for data tables (Dowle and Srinivasan, 2020). data.table is not only faster than other packages implemented in R, but also is significantly more efficient than tools in other languages such as Python and Julia (db-benchmark project, 2021). In addition to speed, data. table has a syntactic structure that is clear and simple to follow. Only three elements are basic to data.table's primary function: $D T\left[i, j, b_{y}\right]$. First, the $i$ section is used to specify the rows to be considered in filtering or subsetting operations. Second, the $j$ section indicates the changes happening in the columns (e.g. adding new ones, changing existing ones). Third, the by section is used to perform operations based on grouping variables. A brief but more exhaustive introduction to data. table can be found in the data. table's vignette and wiki. these alternatives have their own particular advantages. For instance, packages in the tidyverse (e.g. dplyr) are in general designed to increase readability and flexibility during data munging steps (Wickham et al., 2019). Data wrangling in base $\mathrm{R}$ is largely standard and in general more stable over time than other approaches. Here, we focus on extending the functionality of data.table, a package that is generally faster and more concise than other approaches, for dealing with phylogenetic comparative datasets. 
80

81

82

83

84

85

86

87

88

89

90

91

92

93

94

95

96

97

98

99

100

101

102

103

104

105

106

107

108

109

110

111

112

113

114

115

116

117

\section{The treedata.table workflow}

treedata.table is a wrapper for data.table designed for phylogenetic analyses

that matches a phylogeny to a data. table (Table 1). After an initial tree/data

matching step, treedata. table continuously preserves the tree/data matching across data . table operations. treedata. table also allows users to run functions from other phylogenetic packages on the processed treedata . table objects. Below, we briefly explain the general workflow under treedata. table.

1) Tree and character matrix matching: Using the treedata. table package begins with creating a treedata. table object. as.treedata. table function matches the tip.labels of the phylogeny to a column of names in the data.frame.

2) treedata. table operations: two main functions allow users to make changes to treedata. table objects. Changes are reciprocal between trees and data.

A) Explicitly dropping taxa: Taxa in treedata. table objects can be dropped using the droptreedata . table function. Dropped taxa results are removed from the character matrix and trees.

B) Data operations: The most powerful functionality of treedata. table is related to functions calling data.table. The [ function, taking the same arguments as the analog function in data. table, can be used to subset rows, select, and/or compute statistics on columns in the character matrix of the treedata. table object ( $\left.D T\left[i, j, b_{y}\right]\right)$. Operations changing the number of rows in the character matrix will also affect the corresponding taxa in the tree.

3) Data extraction from treedata. table objects: Users can independently extract trees and character matrices from treedata. table objects using the pulltreedata.table function. The \$ operator is also a valid alternative to pulltreedata.table. Two additional functions ( [ [ and extractVectors) can be used to extract named vectors from treedata. table objects. These operations streamline formatting of data into the various different input requirements of $\mathrm{R}$ functions from other phylogenetics packages.

4) Using external functions in treedata. table objects: the tdt function enables users to easily run external functions on treedata . table objects directly. Specifically, tdt passes data and tree attributes from a given 
118

119

120

121

122

123

124

125

126

127

128

129

130

131

132

133

134

135

136

137

138

139

140

141

142

143

144

145

146

147

148

149

150

151

152

153

154

155

156

treedata. table object as arguments to functions implemented in other packages.

5) Additional functions: treedata.tree includes additional functions to detect and filter character matrices by character types (continuous or discrete; detectCharacterType, detectAllCharacters, and filterMatrix). Other functions can be used to examine (head, tail, print) and describe (summary) objects of class treedata. table. Finally, two additional functions can be used to inspect and force column and row names in character matrices (hasNames, forcenames).

\section{Using treedata. table}

This brief step-by-step tutorial is based on treeplyr's Anolis example data, including 100 tips and 11 characters (see also Appendix S1-S2):

library (treedata.table)

data (anolis)

To use all the functionalities in treedata. table, we first construct a

treedata.table object using the as.treedata.table function, which performs an exact name match between the tip labels of the tree and the column in the dataset with the most matches.

td $<-$ as.treedata.table (tree = anolis\$phy, data = anolis\$dat)

The resulting object can be inspected using the summary (), head (), tail (), and print () functions. For instance, we can see a description of the treedata.tree object using the summary () function:

summary $(t d)$

Next, we can perform data manipulation steps on the resulting treedata. table object. For instance, we can extract the SVL column (snout-vent length) using the \$ function and [ operator, as follows:

td\$dat [, 'SVL']

A named vector of the same trait (SVL) can also be extracted using td [ ["SVL"] ] or extractVector ( $t d$, 'SVL'). However, extractVector further supports 
157 extraction of multiple traits. For instance, the following code will extract two named

158

159

160

161

162

163

164

165

166

167

168

169

170

171

172

173

174

175

176

177

178

179

180

181

182

183

184

185

186

187

188

189

190

191

192

193

194

195

196

vectors: one for SVL and another for ecomorph.

extractVector(td, 'SVL', 'ecomorph')

The real power in treedata.table is in co-indexing the tree and table based on functions from data. table. We can use data.table syntax to subset the treedata.table object and include only the first representative from each ecomorph in the Anolis dataset.

td $[, \operatorname{head}(. \mathrm{SD}, 1), \mathrm{by}=$ "ecomorph"]

We can also subset the Anolis dataset to include a single species per ecomorph and island:

$\operatorname{td}[$, head (.SD, 1$)$, by $=$. (ecomorph, island) $]$

Furthermore, we can create a new variable summarizing SVL+hostility for only Cuban anoles:

td $[$ island $==$ "Cuba", . (Index $=$ SVL + hostility) $]$

While the options for data manipulations are infinite, the matching between the tree and data attributes is always constant. Finally, users can pass data and trees in treedata. table objects as arguments to functions in other packages. For instance, below we use the tdt function in treedata. table to fit a continuous model of trait evolution for SVL in geiger (Harmon et al. 2008; Pennell et al. 2014):

tdt(td, fitcontinuous (phy, extractVector(td, 'SVL'), model="BM") )

All the functions explained above can handle multiple trees. For instance, below we fit the same model of continuous trait evolution on SVL based on a multiPhylo tree for the Anolis dataset:

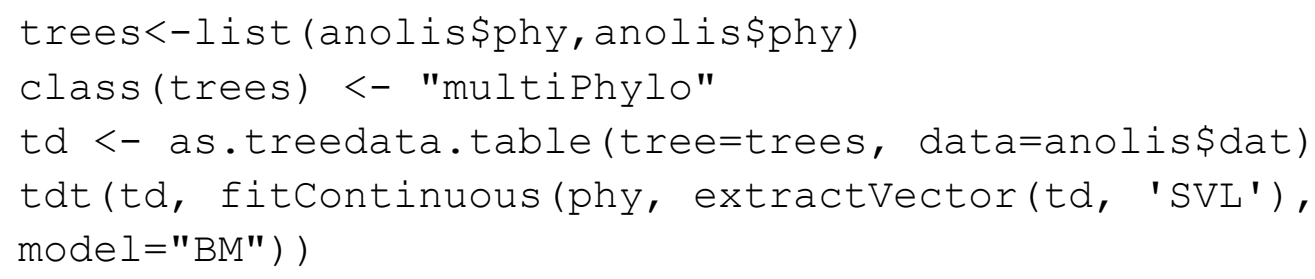


197

198

199

200

201

202

203

204

205

206

207

208

209

210

211

212

213

214

215

216

217

218

219

220

221

222

223

224

225

226

227

228

229

230

231

232

233

234

235

The introductory vignette to treedata.table

(https://ropensci.github.io/treedata.table/articles/AA treedata.table intro english.html, https://ropensci.github.io/treedata.table/articles/AB treedata.table intro spanish.html) contains further information on the functions outlined above and in Table 1.

\section{Computational performance}

Alternatives to treedata. table. Keeping trees and data objects separated in the $\mathrm{R}$ environment is a standard practice. Changes to trees and data are typically performed independently using a combination of functions implemented in ape (Paradis \& Schliep, 2019), base (R Development Core Team, 2013), data . table (Dowle \& Srinivasan, 2019), or in the tidyverse (Wickham et al., 2019). However, to our knowledge, treeplyr (Uyeda \& Harmon, 2020) and tidytree (Yu, 2021), both based on dplyr (Wickham et al., 2019), are to our knowledge, the only packages that are able to perform simultaneous operations on combined tree/data objects in $\mathrm{R}$ (Table 2). We note that while data.table, treeplyr, and dplyr share similar functionalities, their philosophy and syntax are strikingly different. Differences between these packages ultimately relate to "source" package they rely on (i.e. data.table or dplyr). For instance, although data.table uses shorter syntax relative to dplyr, the pipe operator and the use of verbs in dplyr makes this later package more intuitive and easier to debug. Therefore, although our package treedata. table only extends the functionality of data.tables into the phylogenetic comparative workflow, this largely unexplored framework in the field will enable users to take advantage the speed and syntax of that is inherent to data. table.

Methods. We used the microbenchmark (Mersman, 2019) function under default parameters to compare the performance of functions in treedata. table to other packages (Appendix S3). First, we compared the performance in the initial tree/data matching step between treedata. table and treeplyr

(treedata.table: :as.treedata.table() and treeplyr: : make.treedata ( ) ). We simulated trees with 10, 40, and 100 tips using rtree function in ape (Paradis \& Schliep, 2019). Additionally, we generated random character matrices (50 discrete and 50 continuous traits) matching $90 \%$ of tips in the tree. Second, we compared the performance of data operations in treedata. table relative to data . table (Dowle \& Srinivasan, 2019), base (R Development Core Team, 2013), treeplyr (Uyeda 2020), and dplyr (Wickham et al., 2019). This time, we simulated trees with 1000, 10000, and 500000 tips using the rtree function in ape. Again, we generated random character matrices (50 discrete and 50 continuous traits) matching $90 \%$ of tips in simulated trees. We compared the performance of 
treedata.table:: [, data.table:: [ treeplyr:: $>\circ$, dplyr:: $\%$, and

237 the equivalent functions in base when (1) subsampling the full dataset for rows

238 matching a single level in one discrete character, and (2) estimating the sum and mean

239 of two continuous traits based on the groups of a second discrete character. In

240 data. table syntax for this process would be:

241

242

243

244

245

246

247

248

249

250

251

252

253

254

255

256

257

258

259

260

261

262

263

264

265

266

267

268

269

td\$dat $[$ Disc1 $==" A ", .(\operatorname{sum}(\operatorname{Cont} 2), \operatorname{mean}(\operatorname{Cont} 3))$, by $=$ Disc10 $]$

Results. treedata. table was $>400 \%$ faster than treeplyr during the initial data/tree matching step (Fig. 2). For instance, combining a dataset with 10 tips to a character matrix of 40 traits (10\% of unmatched tips), as.treedata. table takes an average of $12.314 \mathrm{~ns}$ (range $=8.100-27.479 \mathrm{~ns}$ ) relative to the $64.198 \mathrm{~ns}$ that were needed in treeplyr (range $=48.407-166.328 \mathrm{~ns}$ ). Differences in the performance between these two functions also scale with the number of taxa. Next, we examined the performance of data operations in treedata. table relative to data. table, base, treeplyr, and dplyr (Fig. 3). We found that the simultaneous processing of phylogenetic trees in treedata.table's compromised the speed of our package by $90 \%$ relative to data. table. However, data manipulation in treedata. table (which simultaneously processes phylogenies) is still significantly faster than in other commonly used packages for data manipulation only, such as base ( $>35 \%)$, treeplyr (>60\%), and dplyr $(>90 \%)$. The higher speed performance of treedata. table relative to other functions also increases with the size of the dataset.

\section{Current limitations of treedata. table}

The current release of treedata. table can handle phylo and multiPhylo objects. A single character matrix is shared across all the trees in the treedata. table object. Additionally, all the trees and the only character matrix in the same treedata. table object are forced to have the same tip-level sampling. We acknowledge that partial tree/data matching is desirable in some situations. For instance, users may be interested in performing analyses on trees that, despite having different tip-level sampling, partially overlap with a common character matrix. Similarly, users may be interested in using multiple character matrices instead of only one. Future releases of the treedata. table package will focus on relaxing some restrictions on the tree/data matching.

270

271

Conclusions

272 Here we describe the first $\mathrm{R}$ package that extends the functionality and syntax of 273 data.table for performing operations in phylogenetic comparative datasets. We also 274 note that treedata. table significantly improves the speed of the analytical workflow 
275 when compared to alternative methods for manipulating phylogenetic comparative data.

276 treedata.table is expected to increase code reproducibility while simplifying the

277 complexity of scripts. Finally, data manipulation in treedata. table, which is

278 significantly faster than in other commonly used packages, will allow researchers to

279 quickly perform data manipulation on large datasets without requiring outstanding

280 computational resources.

281

282

\section{Acknowledgments}

283 We thank Luke Harmon for his contributions that laid the groundwork for the current package. The authors thank Hugo Gruson, Kari Norman, Julia Gustavsen, Luna L. Sanchéz, and Guangchuang Yu for helpful comments during review in rOpenSci and PeerJ. Heidi E. Steiner revised an early version of the manuscript and assisted with logo design.

288

289

\section{Funding Statement}

290

This package was partially developed during the "Nantucket phylogeny developeR

291 workshop", organized by Liam J. Revell (NSF DBI-1759940). AMW was supported by

292

293 an Institutional Development Award (IDeA) from the National Institute of General

294 Medical Sciences of the National Institutes of Health under grant number P2O GM103424-18. JCU was funded on NSF DEB-1208912 to Luke Harmon in creating treeplyr, which served as a precursor for this project and code.

296

297

\section{Authors' contributions}

298

AMW and JCU conceived the project. CRP, AMW, and JCU developed the methods.

299 CRP analyzed the data and led the writing. All co-authors assisted with edits and

300 approved publication.

301

302

303

\section{Data accessibility}

The treedata.table package and documentation are hosted at https://github.com/ropensci/treedata.table.

305

306

\section{Supporting information}

307

Appendix S1. Getting started with the treedata. table package.

308

Appendix S2. Breve introducción al paquete treedata.table.

309

Appendix S3. R script used to perform performance comparison between

310 treedata.table and other methods.

Appendix S4. R script used to generate Figure 1.

312

\section{References}


314 db-benchmark project (2021). Database-like ops benchmark. Available at

315 https://h2oai.github.io/db-benchmark/ (accessed September 13, 2021).

316 Dowle, M., \& Srinivasan, A. (2019). data.table: Extension of 'data.frame'. R package

317 version 1.12.6. https://CRAN.R-project.org/package=data.table (accessed 26

318 August 2020).

319

320

321

322

323

324

325

326

327

328

329

330

331

332

333

334

335

336

337

338

339

340

341

342

343

344

345

346

347

348

349

350

351

352

353

Fitzjohn, R.G., Pennell, M.W., Zanne, A.E., Stevens, P.F., Tank, D.C., \& Cornwell, W.K. (2014). How much of the world is woody? Journal of Ecology, 102, 1266-1272.

Harmon, L. J., Weir, J. T., Brock, C. D., Glor, R. E., \& Challenger, W. (2008). GEIGER: investigating evolutionary radiations. Bioinformatics, 24(1), 129-131.

Harmon, L.J. 2019. Phylogenetic Comparative Methods. No commercial publisher.

Katoh, K. (2002). MAFFT: a novel method for rapid multiple sequence alignment based on fast Fourier transform. Nucleic Acids Research, 30, 3059-3066.

Mcmahon, M.M., Deepak, A., Fernández-Baca, D., Boss, D., \& Sanderson, M.J. (2015). STBase: One Million Species Trees for Comparative Biology. Plos One, 10.

Mersmann, O. (2019). microbenchmark: Accurate Timing Functions. R package version 1.4-7. https://CRAN.R-project.org/package=microbenchmark (accessed 26 August 2020).

Michonneau F., Brown J.W., \& Winter D.J. (2016). rotl: an R package to interact with the Open Tree of Life data. Methods in Ecology and Evolution, 7, 1476-1481.

Paradis, E., \& Schliep, K. (2018). ape 5.0: an environment for modern phylogenetics and evolutionary analyses in R. Bioinformatics, 35, 526-528.

Pennell, M. W., Eastman, J. M., Slater, G. J., Brown, J. W., Uyeda, J. C., FitzJohn, R. G., et al. (2014). geiger v2. 0: an expanded suite of methods for fitting macroevolutionary models to phylogenetic trees. Bioinformatics, 30(15), 22162218.

Piel, W.H., Donoghue, M.J., Sanderson, M.J., \& Netherlands, L. (2000). TreeBASE: a database of phylogenetic information. In Proceedings of the 2nd International Workshop of Species 2000.

R Core Team (2013). R: A language and environment for statistical computing. R Foundation for Statistical Computing, Vienna, Austria. http://www.R-project.org/ (accessed 26 August 2020).

Redelings, B. Sanchez Reyes, L.L., Cranston, K.A., Allman, J., Holder, M.T., \& McTavish, E.J. (2019). Open Tree of Life Synthetic Tree (Version 12.3). Zenodo. doi: 10.5281/zenodo.3937741

Redelings, B.D., \& Holder, M.T. (2017). A supertree pipeline for summarizing phylogenetic and taxonomic information for millions of species. PeerJ, $\mathbf{5}$.

Smith, S.A., \& Brown, J.W. (2018). Constructing a broadly inclusive seed plant phylogeny. American Journal of Botany, 105, 302-314.

Smith, S.A., \& O'Meara, B.C. (2012). treePL: divergence time estimation using penalized likelihood for large phylogenies. Bioinformatics, 28, 2689-2690. 
354 Smith, S.A., Beaulieu, J.M., Stamatakis, A., \& Donoghue, M.J. (2011). Understanding angiosperm diversification using small and large phylogenetic trees. American Journal of Botany, 98, 404-414.

Stamatakis, A. (2006). RAxML-VI-HPC: maximum likelihood-based phylogenetic analyses with thousands of taxa and mixed models. Bioinformatics, 22, 26882690.

Tamura, K., Battistuzzi, F.U., Billing-Ross, P., Murillo, O., Filipski, A., \& Kumar, S. (2012). Estimating divergence times in large molecular phylogenies. Proceedings of the National Academy of Sciences, 109, 19333-19338.

Uyeda, J.C., \& Harmon, L.J. (2020). treeplyr: 'dplyr' Functionality for Matched Tree and Data Objects. R package version 0.1.9. https://github.com/uyedaj/treeplyr (accessed 26 August 2020).

Wickham, H., Averick, M. , Bryan, J., Chang, W., D'Agostino, L., François, et al. (2019). Welcome to the tidyverse. Journal of Open Source Software, 4:1686.

Wickham, H., François, R., Henry, L., \& Müller, K. (2019). dplyr: A Grammar of Data Manipulation. R package version 0.8.3. https://CRAN.Rproject.org/package=dplyr (accessed 26 August 2020).

Yu, G. (2021). tidytree: A Tidy Tool for Phylogenetic Tree Data Manipulation. R package version 0.3.5. https://CRAN.R-project.org/package=tidytree (accessed 14 September 2021). 


\section{Figure legends}

375 Fig. 1. Temporal change in phylogenetic tree sizes between 1978 and 2020 based on 376927 publications for different animal and plant groups. We used a LOESS smoothing to 377 depict the temporal trend in tree size over time. Data was retrieved from the Open Tree 378 of Life (Redelings et al. 2019) using the rot I R package (Michonneau et al. 2016). A 379 linear regression that accounted for lineage identity indicated the significant increase in 380 tree size over time $\left(R^{2}=0.2077, p<0.001\right)$. The $R$ script used to generate this figure is 381 provided in Appendix S4.

382

Fig. 2. Results for the treedata.table microbenchmark during tree/data matching 384 steps. Estimates of the timing during the tree/data matching steps under treedata.table are shown in relation to treeplyr. We show median and lower/upper quartiles times for the performance of each package. The $\mathrm{R}$ script used to 388 generate this figure is provided in Appendix S3.

Fig. 3. Results for the treedata. table microbenchmark during data manipulation. We compare the performance of treedata. table against data. table, base, treeplyr, and dplyr. We show median and lower/upper quartiles times for the 393 performance of each package. The $\mathrm{R}$ script used to generate this figure is provided in 394 Appendix S3. 
Figure 1

Fig. 1. Temporal change in phylogenetic tree sizes between 1978 and 2020 based on 927 publications for different animal and plant groups.

We used a LOESS smoothing to depict the temporal trend in tree size over time. Data was retrieved from the Open Tree of Life using the rotl R package (Michonneau et al. 2016). A linear regression that accounted for lineage identity indicated the significant increase in tree size over time $\left(R^{2}=0.2077, p<0.001\right)$.

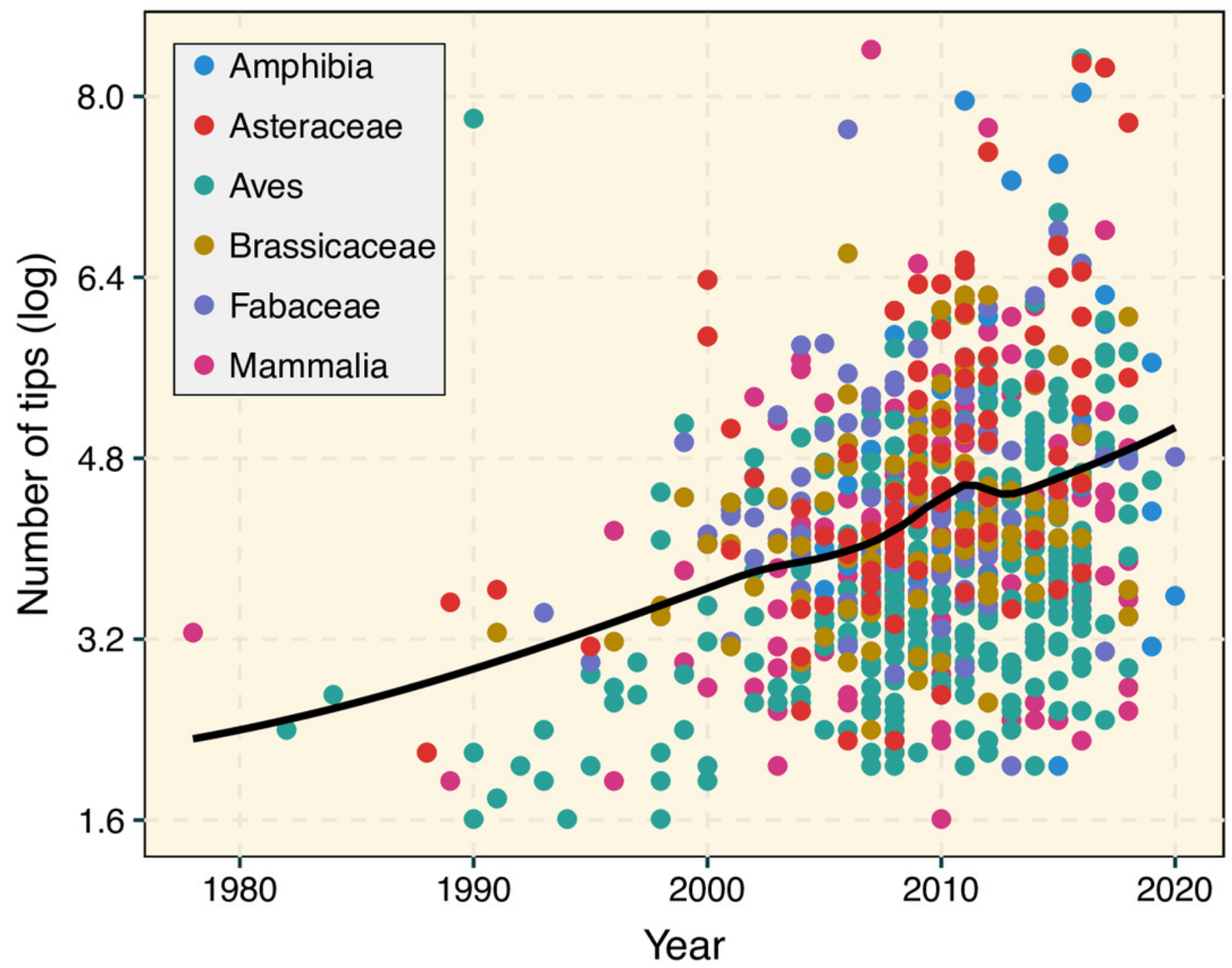


Figure 2

Fig. 2. Results for the treedata.table microbenchmark during tree/data matching steps.

Estimates of the timing during the tree/data matching steps under treedata.table are shown in relation to treeplyr. We show median and lower/upper quartiles times for the performance of each package.
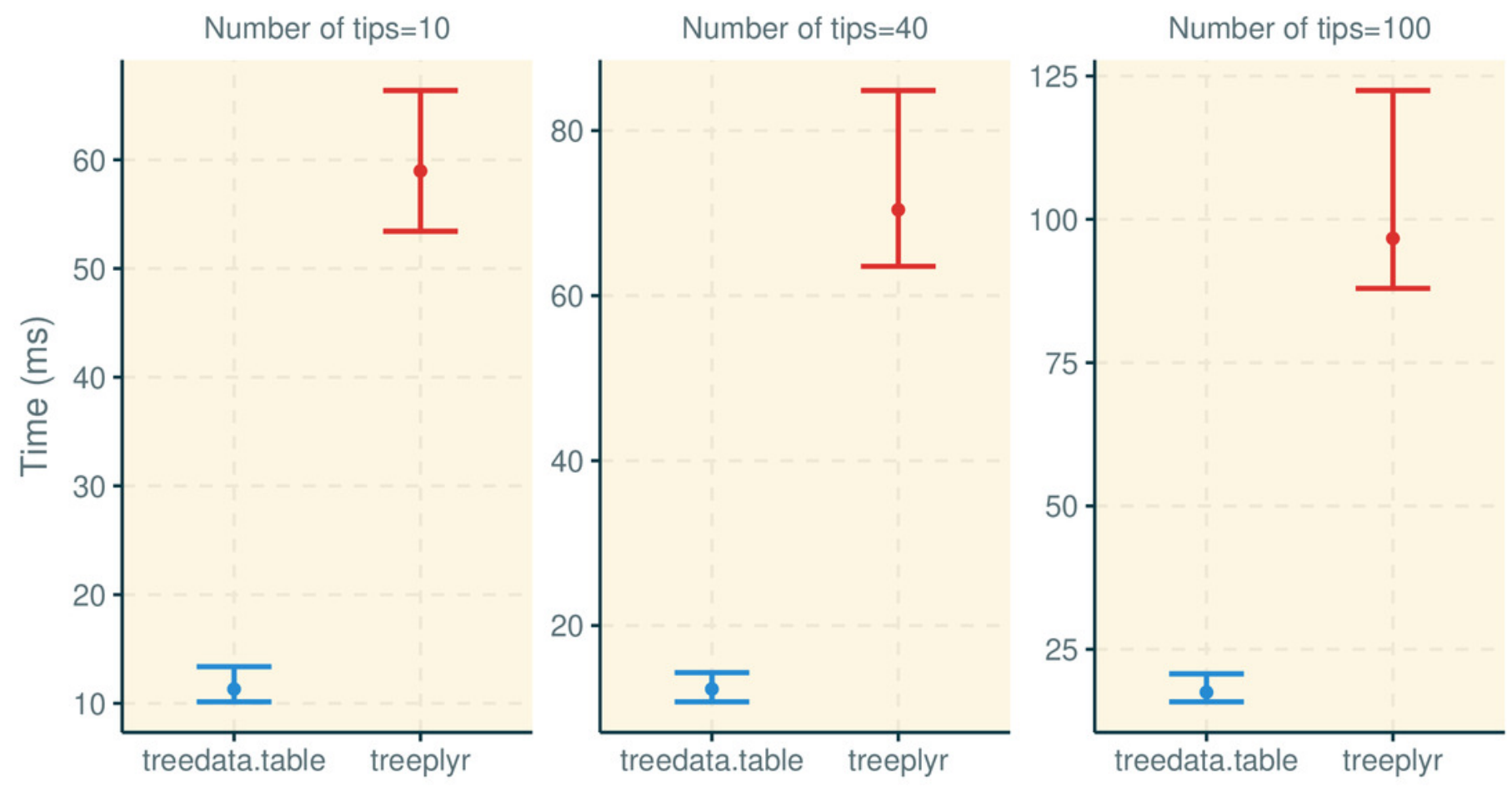
Figure 3

Fig. 3. Results for the treedata.table microbenchmark during data manipulation.

We compare the performance of treedata.table against data.table, base, treeplyr, and dplyr. We show median and lower/upper quartiles times for the performance of each package.
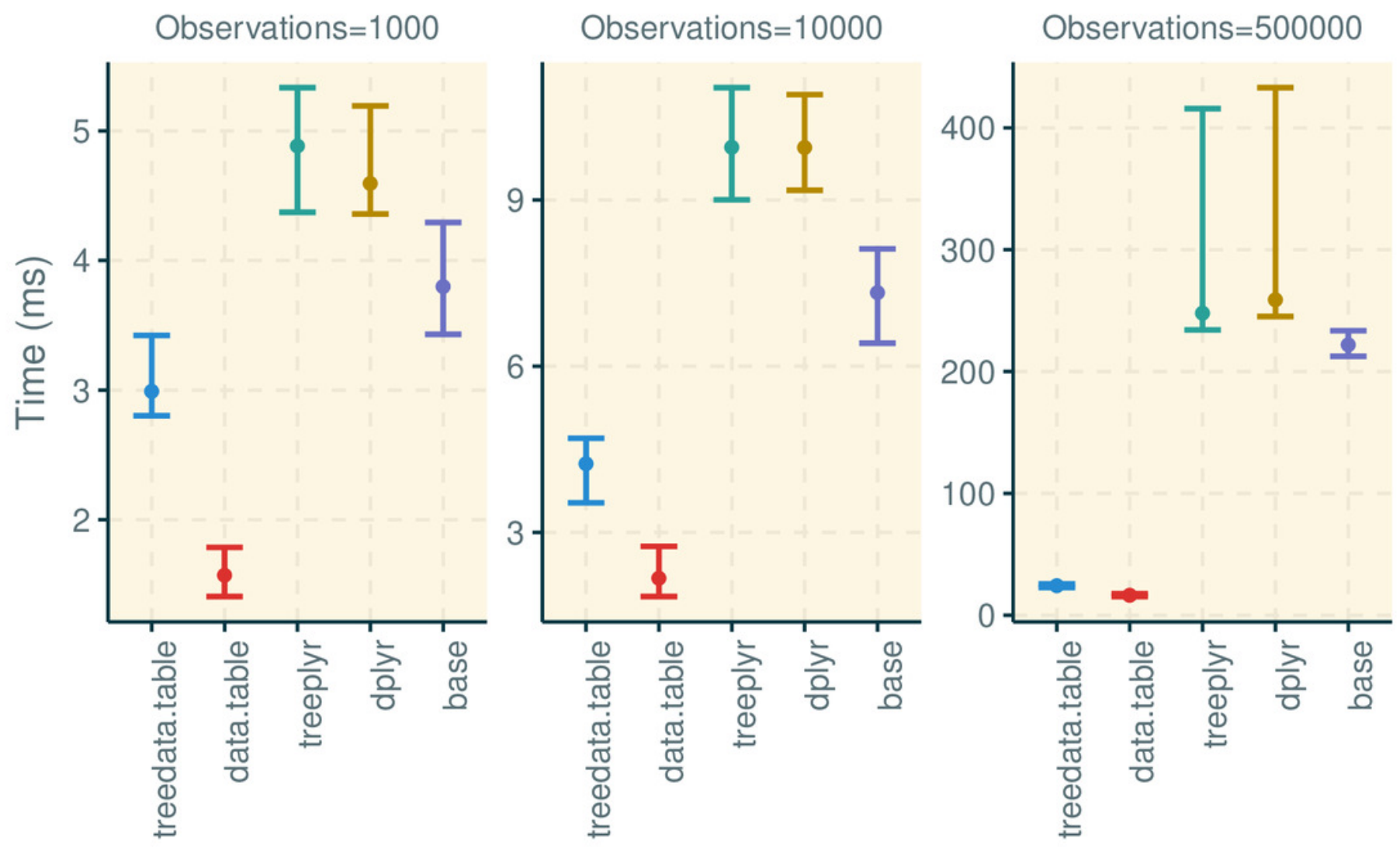


\section{Table $\mathbf{1}$ (on next page)}

Table 1. Brief descriptions of the treedata.table functions. 
1 Table 1. Brief descriptions of the functions implemented in treedata . table

2 functions. We list functions under eight different categories and provide a brief outline of

3 their main uses.

\begin{tabular}{|c|c|c|}
\hline Category & Function & Description \\
\hline $\begin{array}{l}\text { treedata. table object } \\
\text { creation }\end{array}$ & as.treedata.table & $\begin{array}{l}\text { Initial step of the workflow } \\
\text { in treedata. table. } \\
\text { Used to matches a } \\
\text { character matrix (of class } \\
\text { data. frame) to a single } \\
\text { (of class phylo) or } \\
\text { multiple trees (class } \\
\text { multiphylo). }\end{array}$ \\
\hline $\begin{array}{l}\text { Drop taxa from } \\
\text { treedata.table objects }\end{array}$ & droptreedata.table & $\begin{array}{l}\text { Drops taxa from a } \\
\text { treedata.table object }\end{array}$ \\
\hline Data manipulation & {[} & $\begin{array}{l}\text { Function for } \\
\text { performingPerforms } \\
\text { data.table operations } \\
\text { on an object of class } \\
\text {-treedata.table-' }\end{array}$ \\
\hline Data extraction & {$[[$} & $\begin{array}{l}\text { Function for eEx } \text { Etracts a } \\
\text { named vector from an }\end{array}$ \\
\hline
\end{tabular}




\begin{tabular}{|c|c|c|}
\hline & & $\begin{array}{l}\text { object of class } \\
\text { Ltreedata.table' }\end{array}$ \\
\hline & extractVector & $\begin{array}{l}\text { Returning-Returns a } \\
\text { named vector from a } \\
\text { treedata.table object }\end{array}$ \\
\hline & pulltreedata.table & $\begin{array}{l}\text { Returns a character matrix } \\
\text { or tree(s) from a } \\
\text { treedata. table object }\end{array}$ \\
\hline $\begin{array}{l}\text { Run functions from other } \\
\text { packages }\end{array}$ & tdt & $\begin{array}{l}\text { Runs a function on a } \\
\text { Ltreedata.table }{ }^{\prime} \\
\text { object }\end{array}$ \\
\hline \multirow[t]{3}{*}{ Detect character type } & detectCharacterType & $\begin{array}{l}\text { Function to dDetects } \\
\text { whether a character is } \\
\text { continuous or discrete }\end{array}$ \\
\hline & detectAllcharacters & $\begin{array}{l}\text { Applies } \\
\text { detectCharacterType } \\
\text { over an entire character } \\
\text { matrix }\end{array}$ \\
\hline & filterMatrix & $\begin{array}{l}\text { Filters a matrix, returning } \\
\text { either all continuous or all }\end{array}$ \\
\hline
\end{tabular}




\begin{tabular}{|c|c|c|}
\hline & & discrete characters \\
\hline \multirow[t]{3}{*}{$\begin{array}{l}\text { Examine } \\
\text { treedata.table objects }\end{array}$} & summary & $\begin{array}{l}\text { Summarizesing } \\
\text { treedata. table objects } \\
\text { by presenting the number } \\
\text { of discrete and continious } \\
\text { characters, missing values, } \\
\underline{\text { and general changes to the }} \\
\text { original treedata. table } \\
\text { object. }\end{array}$ \\
\hline & print & $\begin{array}{l}\text { Print method } \\
\text { treedata. table objects }\end{array}$ \\
\hline & head, tail & $\begin{array}{l}\text { Returns the first or last part } \\
\text { of an treedata. table } \\
\text { object }\end{array}$ \\
\hline \multirow[t]{2}{*}{ Inspect column/row names } & hasNames & $\begin{array}{l}\text { Row and column name } \\
\text { check }\end{array}$ \\
\hline & forceNames & $\begin{array}{l}\text { Force names for rows, } \\
\text { columns, or both }\end{array}$ \\
\hline
\end{tabular}

4 


\section{Table 2 (on next page)}

Table 2

Functions in different $\mathrm{R}$ packages (including treedata.table) with similar functions on matched tree/data objects. 
1 Table 2. Functions in different $R$ packages (including treedata . table) with similar

2 functions on matched tree/data objects.

\begin{tabular}{|l|l|l|l|}
\hline Package & Function & $\begin{array}{l}\text { Tree/data-matched object } \\
\text { manipulation }\end{array}$ & Reference \\
\hline treedata.table & as.treedata.table & data.table syntax & This study \\
\hline geiger & treedata & Not supported & $\begin{array}{l}\text { Harmon } \\
\text { et al. } \\
(2008), \\
\text { Pennell et } \\
\text { al. (2014) }\end{array}$ \\
\hline tidytree & treedata & $\begin{array}{l}\text { (plyr verbs after using } \\
\text { tibble: :as_tibble () }\end{array}$ & (2021) \\
\hline treeplyr & make.treedata & dplyr verbs & $\begin{array}{l}\text { Uyeda \& } \\
\text { Harmon } \\
(2020)\end{array}$ \\
\hline
\end{tabular}

3

4 